\title{
Accelerated Bearing Life-time Test Rig Development for Low Speed Data Acquisition
}

\author{
A. Klausen ${ }^{1}$ R.W. Folger ${ }^{1}$ K.G. Robbersmyr ${ }^{1}$ H.R. Karimi ${ }^{2}$ \\ ${ }^{1}$ Department of Engineering Sciences, University of Agder, N-4898 Grimstad, Norway. \\ E-mails: andreas.klausen@uia.no, kjell.g.robbersmyr@uia.no, roy.w.folgero@uia.no \\ ${ }^{2}$ Department of Mechanical Engineering, Politecnico di Milano, Via La Masa 1, 20156 Milan, Italy. \\ E-mail: hamidreza.karimi@polimi.it
}

\begin{abstract}
Condition monitoring plays an important role in rotating machinery to ensure reliability of the equipment, and to detect fault conditions at an early stage. Although health monitoring methodologies have been thoroughly developed for rotating machinery, low-speed conditions often pose a challenge due to the low signal-to-noise ratio. To this aim, sophisticated algorithms that reduce noise and highlight the bearing faults are necessary to accurately diagnose machines undergoing this condition. In the development phase, sensor data from a healthy and damaged bearing rotating at low-speed is required to verify the performance of such algorithms. A test rig for performing accelerated life-time testing of small rolling element bearings is designed to collect necessary sensor data. Heavy loads at high-speed conditions are applied to the test bearing to wear it out fast. Sensor data is collected in intervals during the test to capture the degeneration features. The main objective of this paper is to provide a detailed overview for the development and analysis of this test rig. A case study with experimental vibration data is also presented to illustrate the efficacy of the developed test rig.
\end{abstract}

Keywords: Condition Monitoring and Bearing and Low-speed Machinery and Fault Diagnosis and Test Rig

\section{Introduction}

Rolling element bearings, or bearings for short, are necessary in rotating machinery to reduce the degree of freedom of moving parts. A typical bearing is made of an inner-race fastened to the shaft, a stationary outerrace, and rollers in between that transfers the shaft load. The relative distance between each roller is kept constant by a cage. Bearings are precisely manufactured to withstand the dynamic loads acting on the shaft. Metal-to-metal contact is reduced to a minimum by lubricating the bearing with either oil or grease. However, wear will always be present, even in perfectly lubricated bearings. As a roller moves in and out of the radial load zone, the local lubrication is pressurized and causes stress to the rollers and the raceways. After millions of rotations, this cyclic load wears out the bearing components, and cause single or multiple faults which can occur in four different locations: the rollers, the inner-race, the outer-race, and the cage; although cage failures are uncommon. A worn bearing has increased friction, which in turn increases the machine temperature, noise, and vibration levels. A completely worn bearing could cause total system breakdown, injuries, and costly downtime.

It is important to monitor the condition of critical components, such as bearings, to schedule maintenance when necessary and avoid breakdowns. This is especially important for machines operating offshore, like windmill farms (Kandukuri et al., 2016), as they are 
difficult to access and typically has a low profit margin. For bearing condition monitoring, the vibration signal is broadly accepted as a reliable data source. When a roller hits a fault, the impact energy causes the bearing to vibrate at its resonance frequency. This vibration is measured using an accelerometer, and is analyzed to determine the bearing condition. However, bearings faults of low-speed machines are difficult to diagnose as the fault impact energy is lowered, resulting in a low signal-to-noise ratio (SNR). Algorithms that reduce noise and highlight low-energy impacts are important to correctly diagnose a machine operating at low speed. Noise reduction examples in literature are autoregressive model filters (Junsheng et al., 2006), adaptive filters (Antoni and Randall, 2004), and neural networks filters (Zarei et al., 2014), for instance. Highlighting low-energy impacts can also be accomplished with a bandpass filter at the estimated bearing resonance frequency using the fast Kurtogram (Antoni, 2007), or blindly deconvolve the raw signal to preserve parts with high kurtosis (Peled et al., 2005). Analyzing proximity data to diagnose a faulty bearing has also been explored in (Shakya et al., 2016) where the authors state that a proximity sensor is more sensitive than accelerometers during low-speed conditions. Acoustic emission sensors have also gained prominence in the past decade, promising better sensitivity compared to accelerometers during low-speed conditions. Apart from the sensor type selection, low-speed bearing sensor data is necessary to assess the diagnostic accuracy of a newly developed algorithm. Sensors can be used to acquire test data, and they can be mounted on industrial machinery in use, or on specialized test rigs in research facilities. The latter setup type is the most ideal for research as it grants full control of the test and the environment of the bearings. However, for verification purposes, it may be advantageous to test algorithms on industrial machinery as well.

In the literature, different bearing test rigs have been used to generate signal data. Some designs (Sawalhi and Randall, 2008; Kumar and Singh, 2013; Siegel et al., 2012; Sawalhi and Randall, 2011; Niknam et al., 2013) combine a torque source and a test bearing that is monitored for faults. These rigs are unable to apply heavy loads to the bearing, and are thus unable to naturally wear out the bearing within a feasible amount of time. Other test benches also include heavy load capabilities (Lin et al., 2013; Jamaludin et al., 2001), but due to a low rotating speed, the bearing did not wear out naturally. In these cases, the test bearing is installed with an artificially introduced, pre-seed fault, often shaped like a hole or a line. Comparing sensor data from a healthy and a damaged bearing is a typical scenario for testing a diagnostic algorithm.
One missing feature is the ability to follow the development of faults as it would happen in a real scenario. Remaining useful life prognostics of a machine is often dependent on the changes in sensor data as the amount of wear increases. To capture this trend, the bearing may be run to failure on an accelerated life-time test rig. Their designs include equipment for applying loads targeting the test bearing to accelerate its lifetime. The test rigs in (Fan et al., 2015; Muruganatham et al., 2013; Ali et al., 2015; Zhang et al., 2011; Yu, 2011; Li et al., 2012; Danielsen et al., 2017; Qiu et al., 2006) combine high speed and heavy load to achieve faults in a reasonable amount of time. Unfortunately, their equipment does not handle low-speed scenarios for logging data. Some other designs have the loading capacity to wear out the bearing even during low-speed scenarios (Caesarendra et al., 2013; Elforjani and Mba, 2010), using only axial load.

The presented research gives a detailed overview of the development of a new test rig used to acquire lowspeed sensor data. From the literature review, the adopted methodology for this rig is to accelerate the bearing life-time using heavy axial and radial loads at medium-to-high shaft speed. Sensor data is acquired in intervals during the test at lower speeds, reaching down to 20 revolutions per minute (rpm). Required hardware and software design for performing the test is also presented.

The rest of this paper is organized as follows. Section 2 describes all specifications for the test rig, and the different solutions that fulfill these requirements. A combination of solutions are chosen and merged into a final design in Section 3. Additionally, a case study from an accelerated life-time test is also presented. Finally, conclusions are drawn in Section 4.

\section{Methods}

In this section, a short description of accelerated lifetime testing is first given. Then, specifications for the test rig are provided, and possible solutions to fulfill them are discussed.

\subsection{Accelerated life-time testing}

Accelerated life-time testing (ALT) is the process of speeding up the degradation of a component by overloading it to uncover faults in a short amount of time. The test rig described in this paper is designed to drastically reduce the lifetime of a bearing from several years to a few weeks. Subjecting the test bearing with large forces reduces the number of revolutions a bear- 
ing will hold. This comes from the following:

$$
L_{10}=\left(\frac{C}{P}\right)^{3},
$$

where $L_{10}$ denotes the bearing life-time in million revolutions with a $90 \%$ confidence (hence the lower case 10), $C$ is the dynamic capacity of the bearing, and $P$ is the weighted sum of radial and axial loads in Newtons. By running the bearing with a heavy load at a high speed, the life-time is accelerated to uncover faults relatively fast.

\subsection{Specifications}

Before designing the test rig, it is important to set up specifications describing the main features. Further development of concepts is based on these specifications:

1. The driving motor must be able to rotate the shaft at medium-to-high speed to accelerate the lifetime of the bearing, and at a low speed (about $20 \mathrm{rpm})$.

2. The accessible radial and axial loads must be at least $10 \mathrm{kN}$. The load magnitude must also be easy to change.

3. It must be possible to mount sensors measuring bearing data including: temperature, vibration, shock pulses, acoustic emission, and shaft radial movement.

4. The test bearing housing must be big enough to house a 6008 size rolling element bearing with a $68 \mathrm{~mm}$ outer diameter size.

5. The test rig equipment, software, and controller hardware must be designed for unsupervised 24/7 accelerated life-time testing.

The reason for designing a test rig around a small 6008 size bearing is to minimize the cost, space and complexity, as a larger bearing require heavier loads in addition to stronger support structure. In the next subsection, solutions that fulfill the specifications are presented.

\subsection{Concept Development}

Based on the specifications there are various solutions capable of achieving the desired functions. A function tree, illustrated in Figure 1, visualizes feasible options to achieve the main function which is to accelerate the life-time of a bearing. The main function is split into four sub functions that must be achieved, and for each there are several feasible solutions. The viability of each are discussed, with respect to the specifications, to determine which should be kept in the design phase:

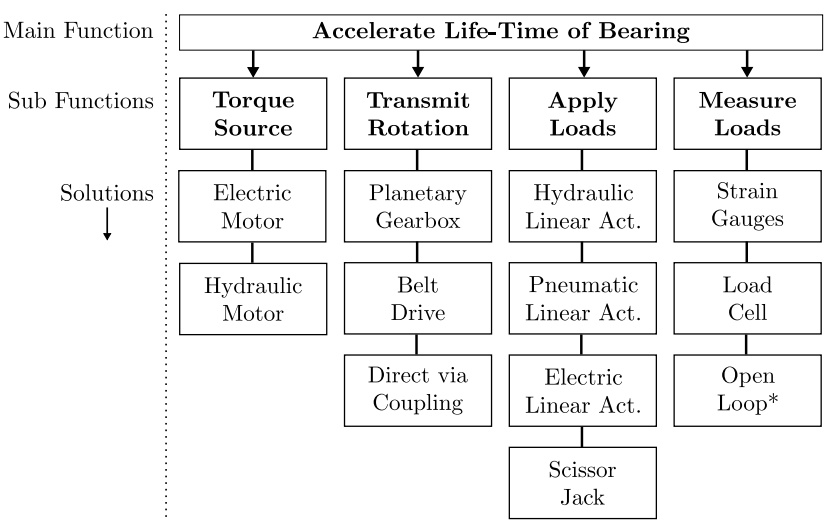

Figure 1: Test rig pre-design function tree. *An OpenLoop force feedback is a feedforward calculation of the assumed forces based on a particular input.

Torque source: It is important that the torque source can control the test bearing shaft at low speed. If the shaft experiences too much unintentional speed fluctuations around the setpoint, the sensor measurements may be hard to analyze. A high-performance motor and frequency drive combination is necessary to fulfill this requirement. In addition, variable speed must also be realizable to simulate some machines undergoing variable conditions, such as winches or windmills. Most motor types have the possibility to be driven at both high and low speed. A hydraulic motor requires an external pressure source to be operational during the entire accelerated life-time test. Using a centralized hydraulic power unit (HPU) in a laboratory for $24 / 7$ operation is not ideal due to power consumption and equipment safety. Installing an HPU for the test rig increase the complexity and cost of parts. Using an electric motor is more ideal since electricity is almost always readily available. The different electric motor types are: brushed DC motor, brush-less DC motor, induction motor, and permanent magnet motor. Brushed DC motors may require more maintenance due to wear of the brushes, and therefore these are not the most suitable. Brush-less DC motors and permanent magnet motors are quite similar in function, as they are synchronous machines. Low-speed operation is necessary, which require an encoder or resolver in the motor. Most induction motors are controlled using sensor-less speed control to reduce cost, but a position-feedback is important for low-speed scenarios to properly control the motor, and log the shaft position during measurements.

Transmit Rotation: The lowest speed requirement is $20 \mathrm{rpm}$, and it is improbable that a motor coupled to the shaft directly will be able to rotate steadily at that low speed. This is because the shaft may be slightly 
unbalanced or bent, resulting in a variable load torque. Without a reasonably high inertia, the motor controller will most likely not react fast enough to the changes in the load torque. Therefore, a gearing ratio of at least 5 is probably necessary to increase the load inertia, and allow the motor to operate at a higher speed. This can be achieved with a belt drive, but a planetary gearbox is more compact to install.

Apply Loads: Using a hydraulic linear actuator (Act.) to load the bearing allows for a high amount of variable force, but require an HPU stationed nearby. Pneumatic linear actuators have some of the same disadvantages, but cannot subject the bearing with high forces due to low permissible air pressure. Hydraulic and pneumatic valves often leak over time, which require the control system to reapply pressure consistently. A scissor jack may create a high force, but varying the load requires manual operation and is not convenient. Electric linear actuators have the advantage that they can self-lock after the desired force is applied, therefore power is only necessary when the load is changing. However, they are often limited by their maximum force. Considering this, a mechanical lever can be used to amplify the load if necessary. It is important to allow for $24 / 7$ operation, and as such it is inconvenient to keep a hydraulic or pneumatic power unit continuously powered on.

Measure Loads: Actuators have losses from friction and power conversions, and these losses may be non-linear, which makes them hard to estimate. Therefore open-loop force calculations will include errors and are thus not ideal to use. Strain gauges can be added to the structure to measure loads with good accuracy. However, such measurements require high-resolution analog-to-digital converters, a model of the system, and a way to calibrate the strain gauge signal based on a known reference. In addition, it may be difficult to suppress unwanted noise due to temperature changes, or to decouple load sources. Off the shelf load cells are pre-calibrated and temperature compensated by the manufacturer, and are thus much easier to use and less error prone.

\section{Results and discussion}

In this section, solutions for the sub functions are chosen and designed. Later, all parts are assembled on a suitable steel rig. The software and electronic hardware designs for performing the accelerated life-time test are elaborated afterwards. Finally, a case-study from an accelerated life-time test using the assembled test rig is presented.

\subsection{The chosen solution}

Based on the discussion of available components in Section 2.3, the following choices are made:

The available electric motor types should all be capable of controlling the shaft at low-speed, assuming that some gearbox is installed, and the controller utilizes a motor position feedback sensor. A permanent magnet motor (PMM) with a high resolution built-in encoder, and a variable frequency drive with a high-performance controller was chosen to drive the shaft. This combination can drive the motor up to $4000 \mathrm{rpm}$, and due to the high efficiency of PMM, it is simples to keep cool during low-speed conditions as squirrel cage induction motor fans typically produce little airflow during this condition.

A planetary gearbox is installed to transmit the rotation torque from the motor to the shaft. It has a gearing ratio of $1: 7$, and is designed to be attached directly to the chosen PMM.

Two electric linear actuators were chosen to produce the radial and axial loads. Each have a loading capacity of $2.5 \mathrm{kN}$, which is too low considering the specifications. To amplify the low force, two levers are designed to amplify them.

Pre-calibrated load cells are used to measure the loads due to difficulties of applying strain-gauges. The chosen cells are shaped as bolts, and can measure the reaction force in a rotational joint up to $20 \mathrm{kN}$. These will be used as hinges for the two levers, and measure the reaction forces at the same time.

\subsection{Test rig design}

The design of the test rig starts with the shaft. The test bearing is installed on one end of the shaft, and the gearbox is connected via a coupling on the other end. Radial and axial loads are applied using linear actuators and levers. Aside from the test bearing, a minimum of two support bearings are also needed to counteract the added radial forces. To avoid wearing out the support bearings, durable ones with higher dynamic capacities compared to the test bearing are necessary. Also, it is important that the support bearings ignore the axial load on the shaft since it must propagate to the test bearing. Radial bearings within split plummer block housings are suitable for these specifications, as this combination give no axial support without installing extra equipment. The axial load must originate from a stationary source, hence a thrust (axial) bearing is installed on the shaft that allow for this load transfer. Four bearings are therefore installed on the shaft: a test bearing, two radial support bearings, and a thrust bearing. Figure 2 (a) shows the acting forces on the shaft, and Figure 2 (b) shows the bearing 


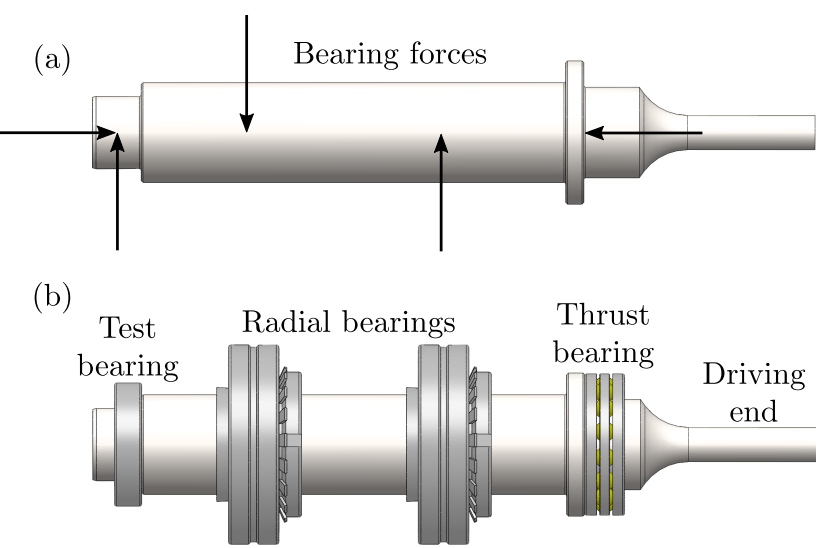

Figure 2: Chosen shaft design and bearing placements.

(a) Counteracting forces on all the bearings.

(b) Position of the required bearings.

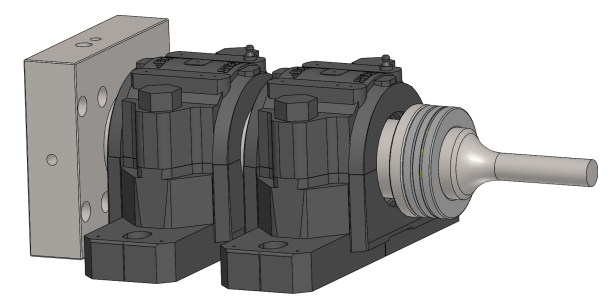

Figure 3: Bearing housings as placed on the shaft.

positions.

Stationary houses are required to transfer load to the rig structure, and to mount sensors. Two suitable split plummer bearing housings are chosen to accommodate the two larger radial bearings. Inside these housings, the two bearings are free to slide in the axial direction. This is critical to transfer the axial load to the test bearing. The test bearing housing is processed in a CNC machine from a steel block. Figure 3 shows the bearing houses on the shaft. The outer area of the test bearing housing can accommodate up to three vibration accelerometers, or shock pulse sensors, via stud mounts. A temperature sensor reaches the outer ring of the bearing via a hole in the housing. An eddy current proximity sensor is stud mounted in a drilled hole in the split plummer bearing housing close to the test bearing. Acoustic emission sensors can be placed using glue on any flat surface on the housing. Figure 4 shows the possible sensor layout on the test bearing housing.

The axial load is added to the shaft via a concentric unit that is placed on the thrust bearing. Its curved area makes the load transfer from the lever easier. Figure 5 shows the concentric unit combined with the thrust bearing. The concentric unit is stationary and acts as a bridge between the lever and the shaft.

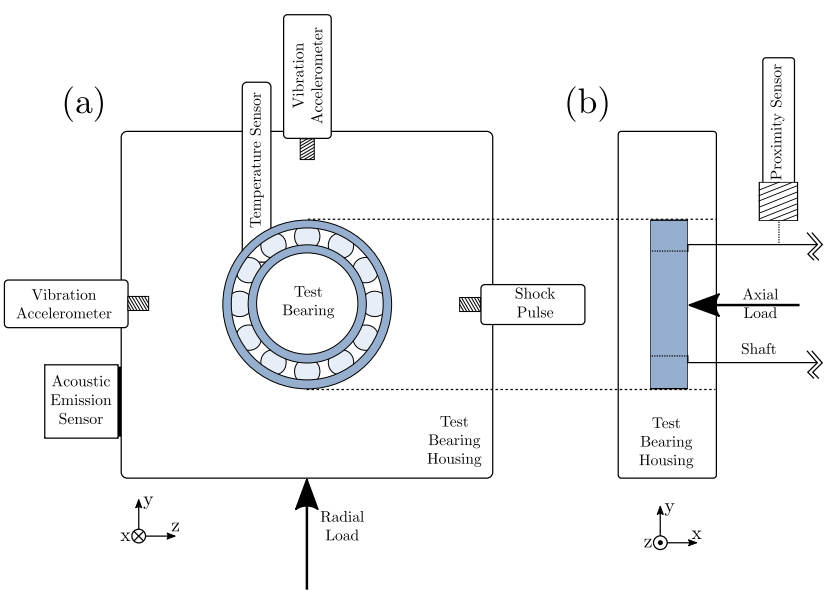

Figure 4: The sensor layout on the test bearing housing: (a) Front of the housing with the bearing in the middle and sensors around it. (b) Side of the test bearing housing with the proximity sensor location.
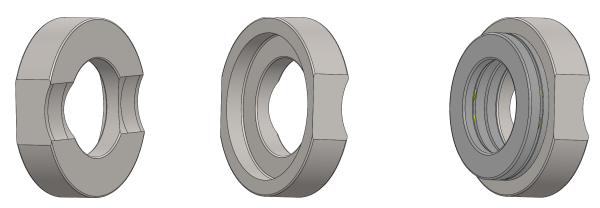

Figure 5: Concentric unit housing the thrust bearing.

From the specifications, at least $10 \mathrm{kN}$ radial and axial loads must be available. Two electric linear actuators, each with a maximum force output of 2.5 $\mathrm{kN}$, are used to apply the loads. Additionally, two levers are designed to amplify each linear actuator force to $17 \mathrm{kN}$. The high amplification was chosen to avoid loading each actuator $100 \%$ to achieve the required force, and to establish some flexibility for testing stronger bearings in the future. Figure 6 shows the lever used to transfer axial load to the shaft. The two lengths $L_{1}$ and $L_{2}$ are determined in such a way that $L_{1} / L_{2}=17 / 2.5=6.8$. The concentric unit is located at the upper end of the lever, and the linear actuator at the bottom. The lever features a curved surface that ensures proper transfer of the force to the concentric unit. The revolution point $\mathrm{A}$ is a load cell bolt that can measure the reaction force in the hinge. The axial force is calculated based on the geometry of the lever and the reaction force feedback from the load cell. Section 3.3 includes more details. The load cell is supported by brackets bolted to the main steel structure. Figure 7 shows the load cell connected to the lever on the axial load setup.

A similar setup is used to produce the radial load which is applied to the test bearing housing from un- 


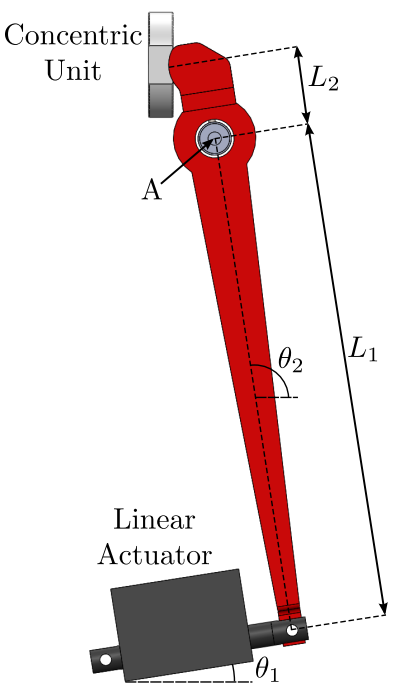

Figure 6: Axial load setup.
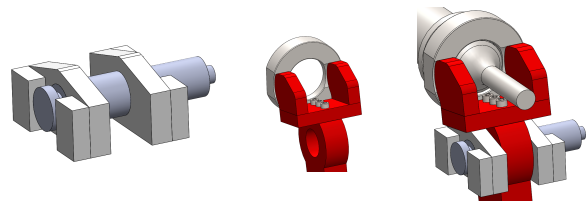

Figure 7: Closeup details of the axial load setup.

derneath. A second linear actuator is connected to a lever which amplifies the radial load. Figure 8 shows the radial load setup. The lever follows the aforementioned geometry ratio to amplify the linear actuator force to $17 \mathrm{kN}$ by making $L_{3} / L_{4}=6.8$. A load cell bolt is used as the revolute joint to measure the reaction force in the hinge. Point B on Figure 8 pushes the bottom of the test bearing housing when the actuator is retracting. The load cell is supported by two brackets as shown in Figure 9. As shown, the lever pushes directly on the bottom of the housing, and the force propagates through the test bearing, to the shaft.

The added axial force propagates through the test bearing, to the test rig structure. The radial force is transferred through the test bearing, to the support bearing housings. Considering this, the test bearing housing require structural support in the axial direction, without interfering in the radial direction. Linear rolling element bearings on rails take care of this behavior. Using four suitable linear bearings between the housing and a support bracket, it is free to move in the vertical direction while supported in the axial direction. Figure 10 shows the housing connected to the linear bearings.

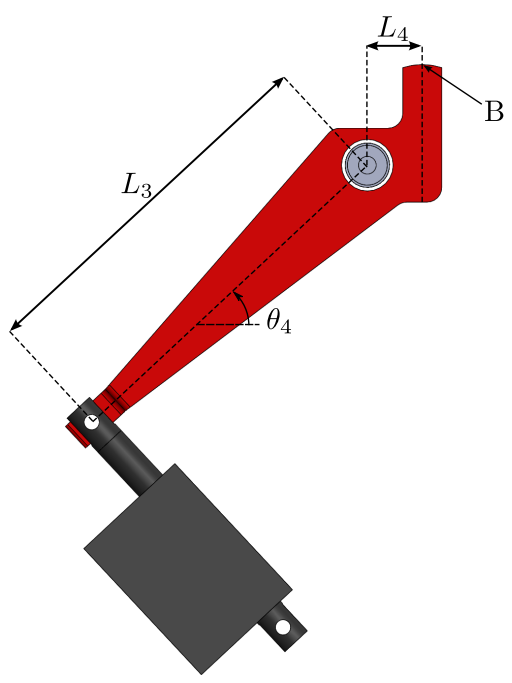

Figure 8: Radial load setup.
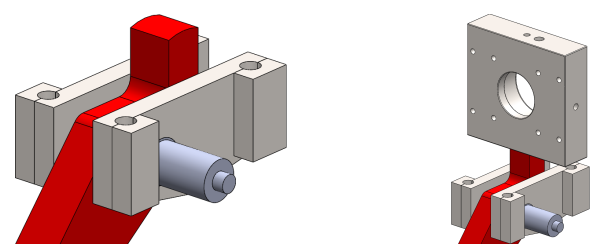

Figure 9: Closeup details of the radial load setup.

\subsection{Axial and Radial Loads}

In the previous subsection, the linear actuator and lever combinations producing axial and radial loads were presented. Load cell bolts are used as lever hinges, and they measure the reaction force in a single direction, as indicated by an arrow on the bolt. The optimal measurement direction must be determined to measure the complete reaction force. During installation, it is important that the load cell orientations are correct. To this aim, the force equilibrium equations are established from a Free Body Diagram (FBD). The FBD of the axial load setup is shown in Figure 11. Here, three forces are acting on the lever, disregarding gravity: $F_{A}$ is the axial force, $R_{1}$ is the load cell reaction force, and $F_{1}$ is the linear actuator force. $\theta_{3}$ is the angular orientation of the load cell necessary to measure the entire reaction force $R_{1}$, i.e. the direction of this force vector. The force and moment equilibrium are given in (2) and (3) respectively.

$$
\sum F=F_{1}\left[\begin{array}{c}
\cos \theta_{1} \\
\sin \theta_{1} \\
0
\end{array}\right]+R_{1}\left[\begin{array}{c}
\cos \theta_{3} \\
\sin \theta_{3} \\
0
\end{array}\right]+\left[\begin{array}{c}
F_{A} \\
0 \\
0
\end{array}\right]=\left[\begin{array}{l}
0 \\
0 \\
0
\end{array}\right]
$$




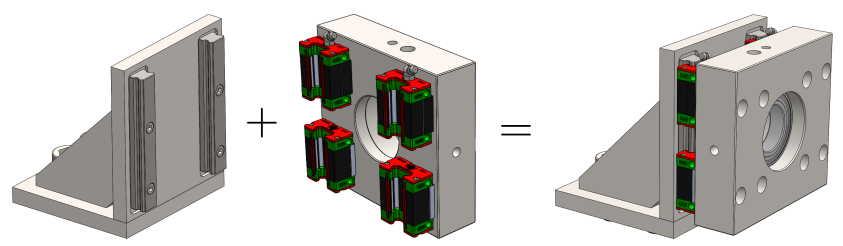

Figure 10: Bracket with linear rolling element bearings connected to the test bearing housing.

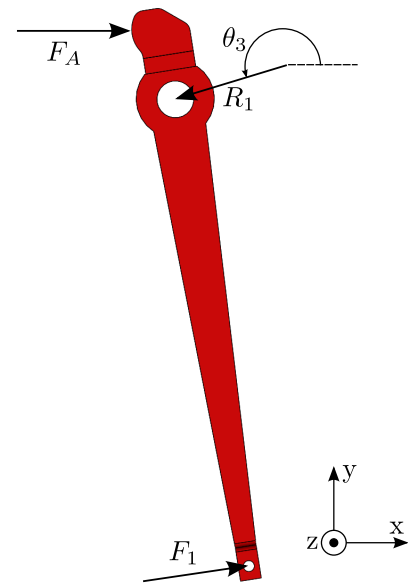

Figure 11: Free Body Diagram of the axial load setup.

$$
\begin{aligned}
\sum M & =L_{1}\left[\begin{array}{c}
-\cos \theta_{2} \\
-\sin \theta_{2} \\
0
\end{array}\right] \times F_{1}\left[\begin{array}{c}
\cos \theta_{1} \\
\sin \theta_{1} \\
0
\end{array}\right] \\
& +L_{2}\left[\begin{array}{c}
\cos \theta_{2} \\
\sin \theta_{2} \\
0
\end{array}\right] \times\left[\begin{array}{c}
F_{A} \\
0 \\
0
\end{array}\right]=\left[\begin{array}{l}
0 \\
0 \\
0
\end{array}\right] .
\end{aligned}
$$

where $\times$ represent the cross product in a right-hand coordinate system, $\sum M$ is the sum of moments, and $\sum F$ is the sum of forces, and the three dimensions are $\mathrm{x}, \mathrm{y}$, and $\mathrm{z}$ respectively. Evaluating (3) yields the following equilibrium in the $\mathrm{z}$-direction

$$
F_{1} L_{1}\left(\sin \theta_{2} \cos \theta_{1}-\cos \theta_{2} \sin \theta_{1}\right)-L_{2} F_{A} \sin \theta_{2}=0 .
$$

Afterwards, (4) is rearranged to isolate the axial force

$$
F_{A}=\frac{F_{1} L_{1}}{L_{2}}\left(\cos \theta_{1}-\frac{\sin \theta_{1}}{\tan \theta_{2}}\right) .
$$

The linear actuator force is isolated from the $y$ dimension of (2)

$$
F_{1}=-R_{1} \frac{\sin \theta_{3}}{\sin \theta_{1}} .
$$

Inserting (6) into (5) yields

$$
F_{A}=\frac{R_{1} L_{1} \sin \theta_{3}}{L_{2}}\left(\frac{1}{\tan \theta_{2}}-\frac{1}{\tan \theta_{1}}\right) .
$$

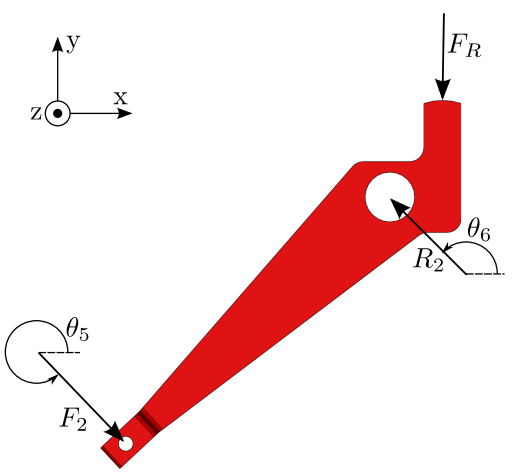

Figure 12: Free Body Diagram of radial load setup.

The $\mathrm{x}$-dimension in (2) is used to determine the hinge reaction force angle

$$
F_{1} \cos \theta_{1}+R_{1} \cos \theta_{3}+F_{A}=0
$$

Inserting (6) and (7) into (8) yields

$$
\begin{aligned}
& -R_{1} \sin \theta_{3} \frac{\cos \theta_{1}}{\sin \theta_{1}}+R_{1} \cos \theta_{3} \\
& +R_{1} \sin \theta_{3} \frac{L_{1}}{L_{2}}\left(\frac{1}{\tan \theta_{2}}-\frac{1}{\tan \theta_{1}}\right)=0 .
\end{aligned}
$$

Afterwards, (9) is rearranged by dividing all terms by $R_{1} \cos \theta_{3}$, and isolating $\theta_{3}$

$$
\theta_{3}=\arctan \left(\frac{1}{\frac{1}{\tan \theta_{1}}+\frac{L_{1}}{L_{2}}\left(\frac{1}{\tan \theta_{1}}-\frac{1}{\tan \theta_{2}}\right)}\right)
$$

$\theta_{3}$ is a constant angle independent of the axial load magnitude, as it is only dependent on the geometry and orientation of the lever. During installation on the test rig, it is important that the rotational orientation of the load cell matches the angle $\theta_{3}$, otherwise the measured reaction force is smaller than in reality. With the load cell in place, $R_{1}$ can be measured and recalculated into $F_{A}$ using (7).

The FBD of the radial load setup is shown in Figure 12. Here, $F_{R}$ is the radial load, $R_{2}$ is the load cell reaction force, $F_{2}$ is the linear actuator force, and $\theta_{6}$ is the angular orientation of the load cell. The force and moment equilibrium for the FBD are given in (11) and (12) respectively.

$$
\sum F=F_{2}\left[\begin{array}{c}
\cos \theta_{5} \\
\sin \theta_{5} \\
0
\end{array}\right]+R_{2}\left[\begin{array}{c}
\cos \theta_{6} \\
\sin \theta_{6} \\
0
\end{array}\right]+\left[\begin{array}{c}
0 \\
-F_{R} \\
0
\end{array}\right]=\left[\begin{array}{l}
0 \\
0 \\
0
\end{array}\right]
$$




$$
\begin{aligned}
\sum M & =L_{3}\left[\begin{array}{c}
-\cos \theta_{4} \\
-\sin \theta_{4} \\
0
\end{array}\right] \times F_{2}\left[\begin{array}{c}
\cos \theta_{5} \\
\sin \theta_{5} \\
0
\end{array}\right] \\
& +\left[\begin{array}{c}
L_{4} \\
0 \\
0
\end{array}\right] \times\left[\begin{array}{c}
0 \\
-F_{R} \\
0
\end{array}\right]=\left[\begin{array}{l}
0 \\
0 \\
0
\end{array}\right]
\end{aligned}
$$

Evaluating (12) yields the following equilibrium in the z-direction,

$$
L_{3} F_{2}\left(\sin \theta_{4} \cos \theta_{5}-\cos \theta_{4} \sin \theta_{5}\right)-L_{4} F_{R}=0 .
$$

Afterwards, (13) is rearranged to isolate $F_{R}$ :

$$
F_{R}=\frac{F_{2} L_{3}}{L_{4}}\left(\sin \theta_{4} \cos \theta_{5}-\cos \theta_{4} \sin \theta_{5}\right) .
$$

The linear actuator force is isolated from the $\mathrm{x}$ dimension in (11) as

$$
F_{2}=-R_{2} \frac{\cos \theta_{6}}{\cos \theta_{5}}
$$

Inserting (15) into (14) yields

$$
F_{R}=\frac{R_{2} \cos \theta_{6} L_{3}}{L_{4}}\left(\cos \theta_{4} \tan \theta_{5}-\sin \theta_{4}\right) \text {. }
$$

The y-dimension in (11),

$$
F_{2} \sin \theta_{5}+R_{2} \sin \theta_{6}-F_{R}=0
$$

is used to determine the hinge reaction force angle. Inserting (15) and (16) into (17) yields

$$
\begin{aligned}
& -\frac{R_{2} \cos \theta_{6}}{\cos \theta_{5}} \sin \theta_{5}+R_{2} \sin \theta_{6} \\
& +\frac{R_{2} \cos \theta_{6} L_{3}}{L_{4}}\left(\sin \theta_{4}-\cos \theta_{4} \tan \theta_{5}\right)=0 .
\end{aligned}
$$

$\theta_{6}$ is isolated in (18) by dividing all terms by $R_{2} \cos \left(\theta_{6}\right)$, and rearranging the result as

$$
\theta_{6}=\arctan \left(\frac{L_{3}}{L_{4}}\left(\cos \theta_{4} \tan \theta_{5}-\sin \theta_{4}\right)-\tan \theta_{5}\right) .
$$

Here, $\theta_{6}$ is a constant angle independent of the radial load, and is only dependent on the geometry and orientation of the lever. After installing the load cell bolt with the orientation given by $\theta_{6}$, the radial force $F_{R}$ is calculated using the measured hinge reaction force in (16).

\subsection{Complete test rig}

Based on the sub function solutions in the previous sections, a complete design of the test rig is created.

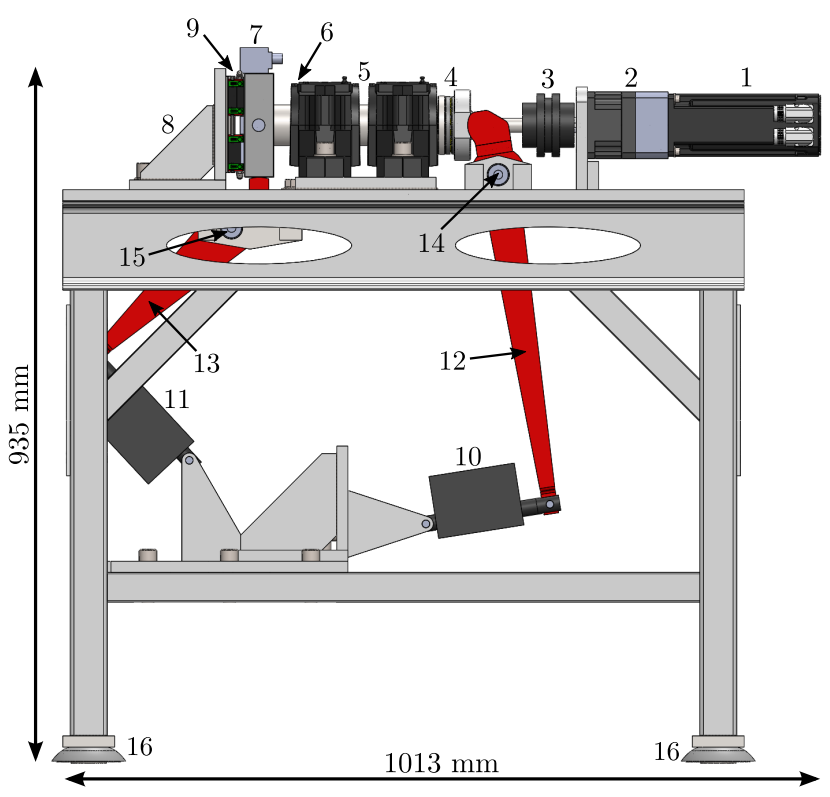

Figure 13: Complete 3D model with numbered components. The width of the test rig is $350 \mathrm{~mm}$.

The chosen solutions achieve the different sub functions; however, the final design shows how the components are placed on a suitable steel structure. The complete test rig is shown in Figure 13. Details of all numbered components are given in the following: To drive the shaft, a permanent magnet synchronous AC motor (1) is chosen. It is powered by a variable frequency drive to realize variable-speed conditions. A 1:7 ratio planetary gearbox (2) is installed to aid the motor during low-speed conditions. This combination enables the shaft to reach speeds up to $500 \mathrm{rpm}$ and down to $20 \mathrm{rpm}$.

A flexible claw coupling (3) that does not transfer axial load is installed to provide shaft rotation. A thrust bearing (4) is installed to transfer axial load to the shaft.

Two bearings (5) are used to counteract the radial load subjected to the test bearing, and they are free to slide in the axial direction inside their housings. On the left end of the shaft at position (6), a proximity sensor is mounted to measure the radial movement of the shaft close to the test bearing.

The test bearing housing (7) is made of steel and is currently manufactured to house a 6008-type ball bearing. Other similarly sized bearings with the same bore diameter may be used, provided that a new housing is created if the outer diameter is different. An accelerometer is placed on the side of the test bearing housing, and the outer ring temperature is measured with a tip sensitive PT100 resistance temperature detector. Other sensors are also available for installation 


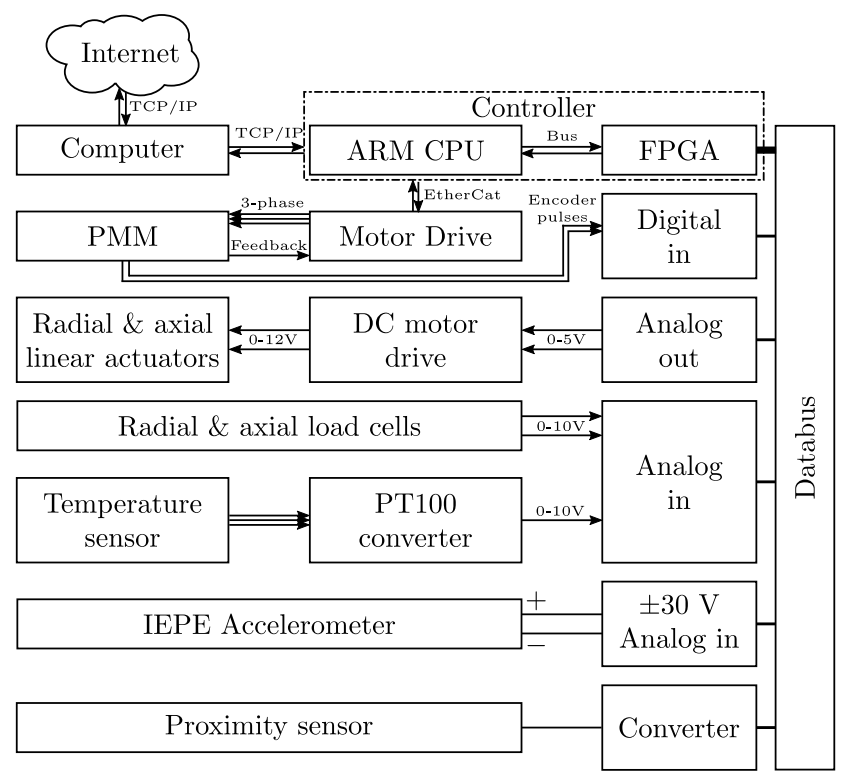

Figure 14: Electronic hardware connections.

as illustrated in Figure 4.

The support bracket (8) is attached to the test bearing housing via linear ball element bearings (9). These bearings allow for vertical load transfer to the test bearing, while they support the shaft in the axial direction. The bracket itself is bolted to the structure.

(10) and (11) are electric linear actuators. They can produce loads up to $2.5 \mathrm{kN}$ in their axial direction. Coupled with the levers (12) and (13), the system can theoretically subject the bearing with $17 \mathrm{kN}$ of load in both axial and radial direction.

Two load cells, (14) and (15), act as hinges for the levers while measuring the respective reaction force up to $20 \mathrm{kN}$.

Machine damping feet (16) are used to reduce the vibration coming from external sources, and their height can also be adjusted to level out the rig on slightly uneven surfaces.

\subsection{Controller hardware}

Electronic hardware is used to control the active components, convert between analog and digital signals, and store sensor data for further processing. A detailed overview of the hardware is shown in Figure 14. A computer with internet access is connected to a dedicated controller with a built-in ARM CPU and Field Programmable Gate Array (FPGA). The main task of the computer is to provide a local and remote interface to the test setup software, and to store data during measurement intervals. A program on the ARM CPU controls all the active components based on the feedback signals and test configuration. The FPGA is re- sponsible for acquiring and sending the analog signals provided by the various $\mathrm{IO}$ via a databus.

The permanent magnet motor (PMM) is controlled by a motor drive with a built-in regulator, and requires only a speed reference from the controller via a real-time EtherCat connection. The shaft angular position is acquired from a quadrature encoder in the motor. The electric linear actuators are powered by a $12 \mathrm{~V}$ DC motor drive, and the reference signal to the drive is between $0-5 \mathrm{~V}$. An input of $0 \mathrm{~V}$ corresponds to full retraction force, $5 \mathrm{~V}$ to full push force, and 2.5 $\mathrm{V}$ for no force. The test-bearing temperature and load cell reaction forces are acquired via an analog-in module. A 24-bit, high-frequency $\pm 30 \mathrm{~V}$ IEPE-compatible analog input module measures the vibration in the accelerometer attached to the test-bearing housing. The accelerometer produces a signal of $100 \mathrm{mV} / \mathrm{g}$ in the linear range up to $10 \mathrm{kHz}$. In addition, a proximity sensor measures the radial shaft movement in one direction. It has a measurement range of $1.1 \mathrm{~mm}$ and a resolution of $18.5 \mathrm{~nm}$, which makes it capable of detecting small changes in shaft position when the bearing is damaged.

\subsection{Load controller design}

The electric linear actuators are controlled to apply the correct radial and axial loads to the test bearing. The choice of control system is not trivial due to several factors: 1) The linear actuator consists of a DC motor connected to a screw joint which moves in and out when the motor rotates. The friction between the screw and DC motor is considerable, and the actuator will therefore self-lock when not active. 2) The recommended duty cycle is less than $10 \%$ to avoid overheating. Specifically, the manufacturer recommends a maximum of 2 minute operation per 20 minutes. 3) Experimental testing on the finished test rig revealed that the measured axial and radial loads varies with the shaft angular position. The variation resembles a sine wave with a certain amplitude if the shaft speed is constant. This variation is most likely caused by asymmetries on the shaft. It may be slightly bent from the manufacturing process, or perhaps not perfectly circular. Due to this disturbance, the actuators are difficult to control in a closed loop.

In any case, care must be taken not to overload the bearing or overheat the actuators. A PID controller would attempt to cancel out the cyclic disturbance from the shaft, risking overshoots and overheating. Using small gains would be a possibility, however that will result in a poor bandwidth.

The self-locking ability is instead exploited to create a simple control system similar to Sliding Mode Control. For each actuator setup, the error $e$ between the reference load $F^{(r e f)}$ and the actual load $F$ is continu- 

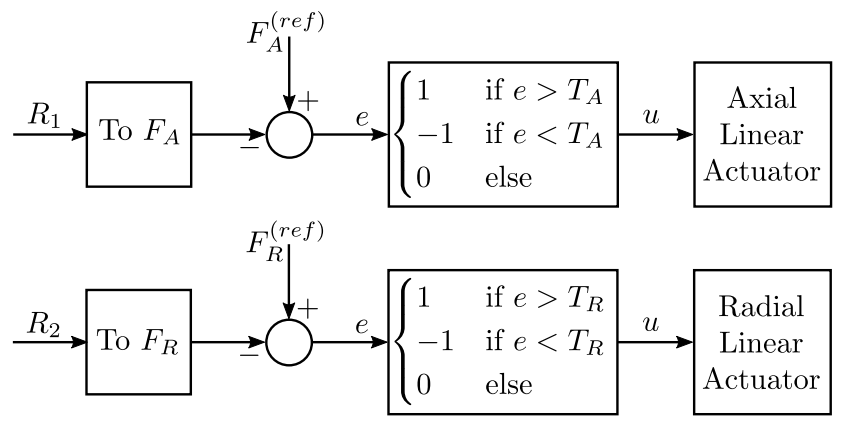

Figure 15: Control scheme for both linear actuators.

ously checked. If this error is outside a threshold $T$, the actuator is activated with maximum power $(u= \pm 1)$ to reduce the error. Once the error is within the threshold, the actuator is deactivated $(u=0)$. By configuring this threshold slightly larger than the amplitude of the shaft disturbance, the actuator is only powered in a short period after load reference change. The control scheme for each actuator load setup is shown in Figure 15 . Here, $R_{1}$ and $R_{2}$ denotes the measured reaction force in the axial and radial load cell, respectively. The block "To $F$ " determines the axial or radial load using Equation (7) or (16), respectively.

It should be noted that this control scheme is not suitable for following a continuously changing reference signal, i.e. a sine wave. For such an input, the controller will actuate the system in steps when the error exceeds the threshold, resulting in a step-wise load change.

\subsection{Accelerated life-time test software design}

An accelerated bearing life-time test may last for several weeks, or even months. Therefore, it is impractical to manually make measurements at a defined interval, or to monitor the test in person. To overcome this, an automated lab test program is developed. This program controls all the active components such as the motor and the linear actuators, and initiates sensor data logging at pre-defined intervals. The flowchart of the test program is shown in Figure 16. Initially, the operator starts by preparing $n_{c}$ configurations (configs for short) that the program runs through. Each config contains specifications for: the shaft speed, bearing load, and the duration. The duration is specified in either number of revolutions (revs) if sensor data is logged, or number of minutes if not. Afterwards, the test may start from the first config, and after it is finished, the next config is loaded and performed. Once all configs are finished, the program resets to the first one.

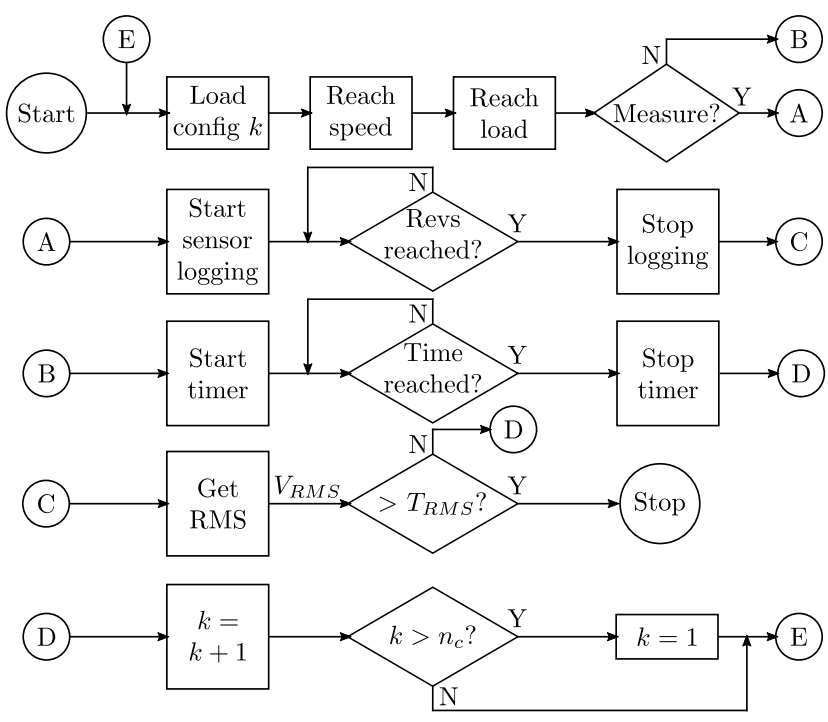

Figure 16: Control scheme for the accelerated life-test.

The test is stopped automatically if the vibration root mean square (RMS) exceeds a preset threshold $T_{R M S}$. The RMS is calculated using

$$
V_{R M S}=\sqrt{\frac{1}{n} \sum_{i=1}^{n} V_{i}^{2}},
$$

where $n$ is the number of samples in the dataset, and $V_{i}$ denotes the $i$ 'th sample in the dataset $V$. Once the RMS reaches the threshold, the bearing is considered completely worn out, and the test is automatically stopped. It is also possible to use different sources for stopping the test such as motor torque or bearing temperature. Additionally, trends of the RMS, motor torque, and bearing temperature is automatically uploaded to a secure folder on the internet, and may be observed remotely by the operator. Further, the test computer may also be accessed via a secure remote desktop connection in case the test must be stopped/changed outside of working hours. Using this setup, the test rig is safe to operate $24 / 7$ without supervision.

\subsection{Case study}

As described in Section 2.1 the test rig is used to perform accelerated life-time tests on bearings. In addition to being able to run at high-speed to degenerate the bearing faster, it also operates at slower speeds to obtain low-speed vibration data. For this accelerated life-time test, the following options are set:

- The shaft is set to run at $500 \mathrm{rpm}$ to wear out the test bearing. 


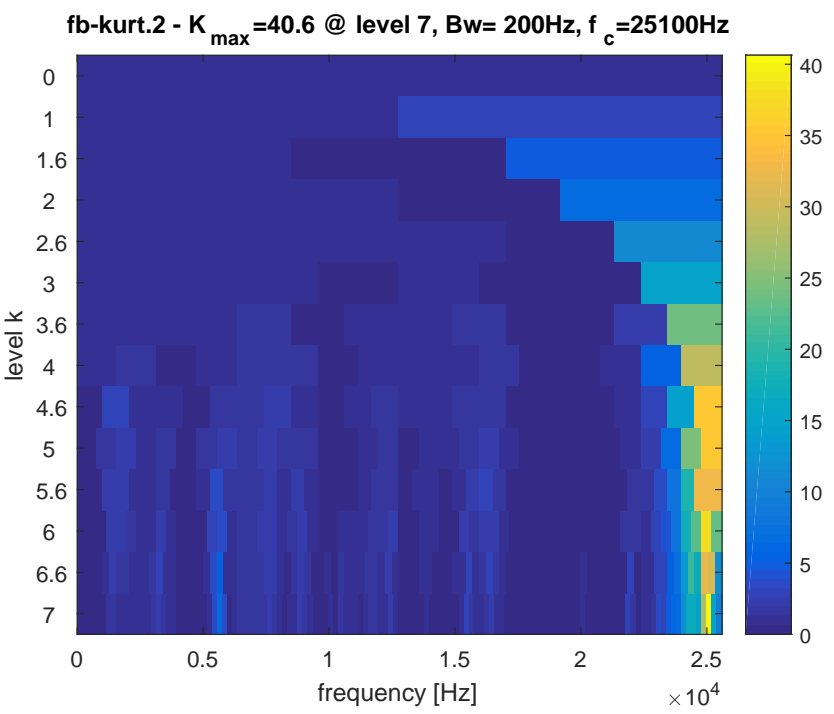

Figure 17: Resulting kurtogram.

Table 1: Operator settings for the test $\left(n_{c}=6\right)$.

\begin{tabular}{llllll}
\hline Conf. & Speed & $F_{A}$ & $F_{R}$ & Rec.? & Duration \\
\hline 1 & $500 \mathrm{rpm}$ & $7 \mathrm{kN}$ & $9 \mathrm{kN}$ & No & $10 \mathrm{~min}$ \\
2 & $500 \mathrm{rpm}$ & $7 \mathrm{kN}$ & $9 \mathrm{kN}$ & Yes & $100 \mathrm{revs}$ \\
3 & $250 \mathrm{rpm}$ & $7 \mathrm{kN}$ & $9 \mathrm{kN}$ & Yes & $100 \mathrm{revs}$ \\
4 & $100 \mathrm{rpm}$ & $7 \mathrm{kN}$ & $9 \mathrm{kN}$ & Yes & $100 \mathrm{revs}$ \\
5 & $50 \mathrm{rpm}$ & $7 \mathrm{kN}$ & $9 \mathrm{kN}$ & Yes & 100 revs \\
6 & $20 \mathrm{rpm}$ & $7 \mathrm{kN}$ & $9 \mathrm{kN}$ & Yes & 100 revs \\
\hline
\end{tabular}

- The radial load is set to $9 \mathrm{kN}$, and the axial load to $7 \mathrm{kN}$. This results in a nominal lifetime of $L_{10}=6$ million revolutions according to the bearing manufacturer's online calculator (SKF, (Link accessible October 2017).

- The bearing vibration is recorded at a sample rate of $51.2 \mathrm{kHz}$, while the bearing temperature, motor torque, and shaft angular position are recorded at a sample rate of $512 \mathrm{~Hz}$.

- Sensor datasets are obtained every 10 minutes at 500, 250, 100, 50, and $20 \mathrm{rpm}$, successively. The measurement duration is set to 100 revolutions.

- The automatic stop was set to an RMS threshold of $3 \mathrm{~g}\left(1 \mathrm{~g}=9.81 \mathrm{~m} / \mathrm{s}^{2}\right)$.

A summary of the configuration loaded into the test software is given in Table 1.

Two weeks and approximately 6 million revolutions later, the bearing is sufficiently damaged that the RMS threshold is triggered. To diagnose the bearing for faults, the datasets acquired at $50 \mathrm{rpm}$ are used on this occasion. To determine the fault condition in
Table 2: Characteristic bearing fault frequencies in shaft orders.

\begin{tabular}{cccc}
\hline BPFI & BPFO & FTF & BSF \\
\hline 6.88 & 5.12 & 0.43 & 3.33 \\
\hline
\end{tabular}

the bearing, a state-of-the-art method named the Fast Kurtogram (Antoni, 2007) is employed. The method decomposes the vibration data into frequency narrowbands at different central frequencies and widths. The kurtosis of each decomposed signal is calculated using

$$
\operatorname{Kurt}\{x\}=\frac{\mu_{4}}{\sigma^{4}},
$$

where $\mu_{4}$ is the fourth central moment, and $\sigma$ is the standard deviation. A high kurtosis value imply an impulsive signal which resembles bearing fault impact vibration. The frequency narrow-band with the highest kurtosis is therefore chosen as the optimal filter, and this band should contain the bearing fault impact vibration. Using this method, the kurtogram is employed at every dataset acquired at $50 \mathrm{rpm}$ shaft speed from the accelerated life-time test. After the signal is filtered using the optimal filter, it should contain the high-frequency resonance vibration from the bearing. To identify the cyclic frequencies in the signal, the signal is demodulated. Using the Hilbert transform, the complex-valued analytic signal is obtained. By computing the absolute value of this analytic signal, the envelope is obtained. The envelope spectrum (ES) is the Fourier transform of this envelope, which contain all cyclic impact frequencies. It has been shown that the envelope should be squared to remove extra peaks in the spectrum (Ho and Randall, 2000), hence resulting in the squared ES (SES). The resulting SES after bandpass filtration is manually analyzed to identify prominent peaks at the characteristic fault frequencies for the present bearing, which are given in Table 2. In this table, BPFI is the ball pass frequency inner race, $\mathrm{BPFO}$ is the ball pass frequency outer race, FTF is the fundamental train frequency, and BSF is the ball spin frequency. One shaft order is defined as the shaft speed, and ball faults are shown at $2 \mathrm{xBSF}$ as there is an impact at the inner and outer race successively during one spin. Equations for calculating these characteristic frequencies are given in (Randall and Antoni, 2011). These characteristic bearing frequencies are of interest because they describe how many times an incipient fault on a certain location is passed on each shaft revolution. The earliest sign of bearing fault is identified after 5.37 million revolutions, and in this case, a ball fault is progressing. The kurtogram is shown in Figure 17, where the width of each frequency band is 


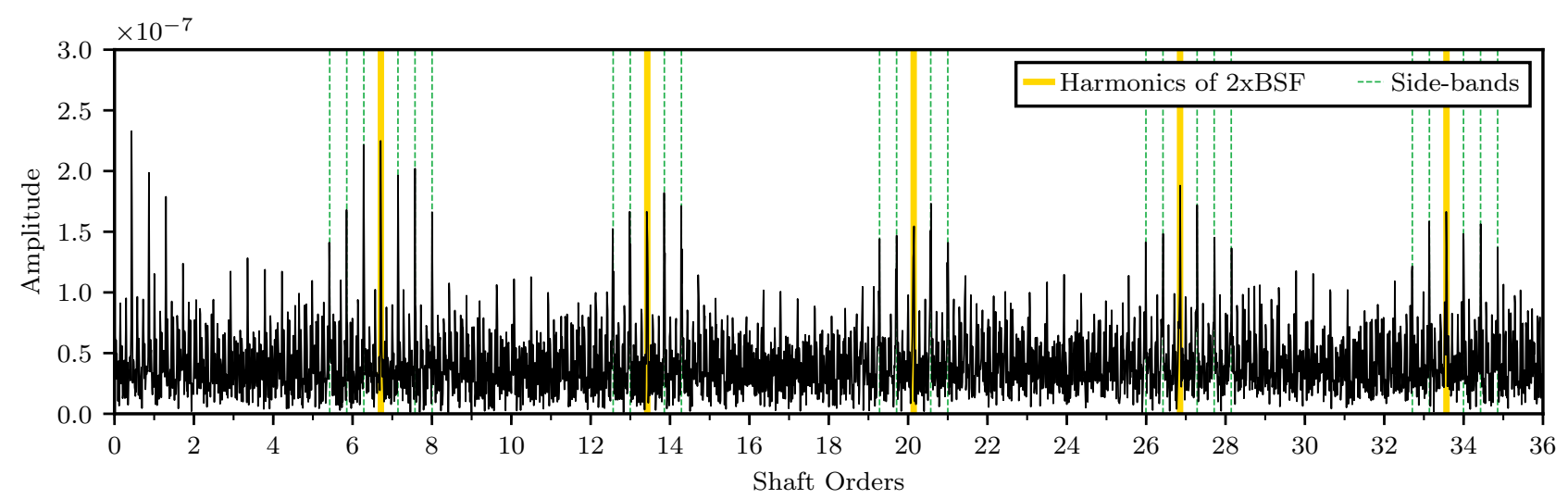

Figure 18: Squared Envelope Spectrum after bandpass filtering.

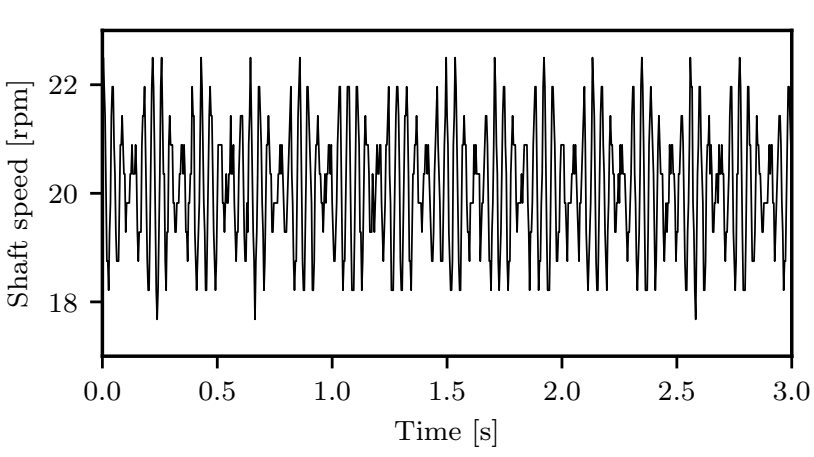

Figure 19: Shaft rotational speed at a reference speed of $20 \mathrm{rpm}$.

given as levels in the $\mathrm{y}$-axis, and the central frequency is given on the $\mathrm{x}$-axis. The kurtosis values are displayed as colors, and the maximum kurtosis of 40.6 is identified at the central frequency $25,100 \mathrm{~Hz}$ with a bandwidth of $200 \mathrm{~Hz}$ (level 7). The resulting SES after band-pass filtering is shown in Figure 18. Here, integer multiples of 2xBSF (harmonics) are marked as yellow lines together with side-bands marked as green stapled lines. The side-bands spaced apart by the FTF are shown due to the amplitude modulation from the nonhomogeneous radial load (Randall and Antoni, 2011). As there are multiple harmonics with accompanying side-bands close to the theoretical $2 \mathrm{xBSF}$, it is likely that the vibration is caused by a ball fault.

The fast Kurtogram filtered SES from a dataset recorded at 20 rom was also analyzed, however it did not show prominent peaks at this low speed. More advanced processing methods are required for diagnosing the bearing during this low-speed working condition. To check if the problem of diagnosing the bearing at $20 \mathrm{rpm}$ is due to speed fluctuations, the shaft speed for the 10 first revolutions is derived from encoder data, and shown in Figure 19. Accordingly, the speed fluc- tuations are within $\pm 10 \%$, which may deteriorate the diagnostic capabilities of the fast Kurtogram slightly. Angular re-sampling should be applied to reduce the blurring effect of the speed fluctuation (Randall and Antoni, 2011).

\subsection{Future work}

In the case study in Section 3.8, it was shown that early fault detection is possible using the fast Kurtogram (Antoni, 2007) at $50 \mathrm{rpm}$, however not at $20 \mathrm{rpm}$. The main purpose of this case study, however, is to demonstrate that the test rig is capable of accelerating the life-time of the bearing, and that the acquired signals may be used for fault diagnosis. Future research will focus on new methods and algorithms for fault detection at the low speed of $20 \mathrm{rpm}$.

After determining the initial fault, it is advisable to predict the remaining useful life-time (RUL) using prognostics methods. These models are important to help schedule maintenance and avoid unexpected breakdowns. Typically, extracted features from sensor data is used to create a prognostic model, and a variety of models and methods have already been suggested in the literature (Peng et al., 2010; Ahmadzadeh and Lundberg, 2014). However, there are still challenges to overcome in designing a standardized scheme that can be applied on any machine (Lee et al., 2014). Additionally, one apparent challenge in rotating machinery prognostics is to make accurate estimations during variable speed or load conditions (Heng et al., 2009).

On the presented test rig, the bearing temperature and vibration, motor torque, and shaft movement is measured for the whole life-span of the bearing during the accelerated life-time test. Further, the working speed of the bearing is also changed between each measurement, and may even be variable during the measurement period. In comparison to bearing test rigs 
relying on pre-seeded faults, accelerated life-time tests allows for tracking the changes in sensor data features as time/wear progresses, making RUL estimation possible. The acquired sensor data will be used in future research to develop algorithms which estimate RUL for machines operating under variable conditions.

\section{Conclusion}

An accelerated life-time test rig for rolling element bearings have been developed in this paper. Using electric actuators and levers, the test bearing can be subjected with heavy radial and axial loads. Additionally, utilizing a variable frequency drive, a motor, and a planetary gearbox, both high and low speed working conditions are realizable. Sensors are installed to measure physical data on the test bearing including vibration, shaft radial movement, and temperature. Tests show that the test rig can be used to generate lowspeed vibration data during all bearing states ranging from healthy to completely worn. Therefore, all the design criteria from Section 2.2 are fulfilled. The list below shows conclusive remarks of the design specifications, where $(\sqrt{ })$ means fulfilled, $(\approx \sqrt{ })$ means partially fulfilled, and $(\times)$ means not fulfilled:

1. $(\sqrt{ })$ - Results show that the low speed of $20 \mathrm{rpm}$ is achievable using a permanent magnet motor and a planetary gearbox. There is a small fluctuation of $\pm 2 \mathrm{rpm}$, which may be corrected using digital angular re-sampling.

2. $(\sqrt{ })$ - Theoretically, the load setups can apply up to $17 \mathrm{kN}$ of load in the radial and axial direction.

3. $(\sqrt{ })$ - The flat edges of the test bearing housing allow for using all the requested sensor types.

4. $(\sqrt{ })$ - The test bearing housing can house the 6008type bearing with $68 \mathrm{~mm}$ outer diameter.

5. $(\sqrt{ })$ - Using suitable hardware and software, the test rig may be operated safely $24 / 7$ without supervision.

\section{Acknowledgements}

This work has been funded by the Ministry of Education and Research in Norway.

\section{References}

Ahmadzadeh, F. and Lundberg, J. Remaining useful life estimation: review. Int. J. Syst. Assur. Eng.
Mang., 2014. 5(4):461-474. doi:10.1007/s13198-0130195-0.

Ali, J. B., Fnaiech, N., Saidi, L., Chebel-Morello, B., and Fnaiech, F. Application of empirical mode decomposition and artificial neural network for automatic bearing fault diagnosis based on vibration signals. Appl Acoust, 2015. 89:16-27. doi:10.1016/j.apacoust.2014.08.016.

Antoni, J. Fast computation of the kurtogram for the detection of transient faults. Mech. Syst. Sig. Process., $2007 . \quad 21(1): 108-124$. doi:10.1016/j.ymssp.2005.12.002.

Antoni, J. and Randall, R. Unsupervised noise cancellation for vibration signals: part I-evaluation of adaptive algorithms. Mech. Syst. Sig. Process., 2004. 18(1):89-101. doi:10.1016/S0888-3270(03)00012-8.

Caesarendra, W., Kosasih, P. B., Tieu, A. K., Moodie, C. A. S., and Choi, B.-K. Condition monitoring of naturally damaged slow speed slewing bearing based on ensemble empirical mode decomposition. J. Mech. Sci. Technol., 2013. 27(8):2253-2262. doi:10.1007/s12206-013-0608-7.

Danielsen, H. K. et al. Multiscale characterization of White Etching Cracks (WEC) in a 100Cr6 bearing from a thrust bearing test rig. Wear, 2017. 370:7382. doi:10.1016/j.wear.2016.11.016.

Elforjani, M. and Mba, D. Accelerated natural fault diagnosis in slow speed bearings with acoustic emission. Eng. Fract. Mech., 2010. 77(1):112-127. doi:10.1016/j.engfracmech.2009.09.016.

Fan, B.-Q., Lee, K.-M., Ouyang, X.-P., and Yang, H.Y. Soft-switchable dual-pi controlled axial loading system for high-speed emu axle-box bearing test rig. IEEE Trans. Ind. Electron., 2015. 62(12):7370-7381. doi:10.1109/TIE.2015.2458303.

Heng, A., Zhang, S., Tan, A. C., and Mathew, J. Rotating machinery prognostics: State of the art, challenges and opportunities. Mech. Syst. Sig. Process., 2009. 23(3):724-739. doi:10.1016/j.ymssp.2008.06.009.

Ho, D. and Randall, R. Optimisation of bearing diagnostic techniques using simulated and actual bearing fault signals. Mechanical systems and signal processing, 2000. 14(5):763-788.

Jamaludin, N., Mba, D., and Bannister, R. Condition monitoring of slow-speed rolling element bearings using stress waves. Proc. Inst. Mech. Eng. Part E J. Process Mech. Eng., 2001. 215(4):245-271. doi:10.1177/095440890121500401. 
Junsheng, C., Dejie, Y., and Yu, Y. A fault diagnosis approach for roller bearings based on emd method and ar model. Mech. Syst. Sig. Process., 2006. 20(2):350-362. doi:10.1016/j.ymssp.2004.11.002.

Kandukuri, S. T., Klausen, A., Karimi, H. R., and Robbersmyr, K. G. A review of diagnostics and prognostics of low-speed machinery towards wind turbine farm-level health management. Renewable Sustainable Energy Rev., 2016. 53:697-708. doi:10.1016/j.rser.2015.08.061.

Kumar, R. and Singh, M. Outer race defect width measurement in taper roller bearing using discrete wavelet transform of vibration signal. Measurement, 2013. 46(1):537-545. doi:10.1016/j.measurement.2012.08.012.

Lee, J., Wu, F., Zhao, W., Ghaffari, M., Liao, L., and Siegel, D. Prognostics and health management design for rotary machinery systems-Reviews, methodology and applications. Mech. Syst. Sig. Process., 2014. 42(1):314-334. doi:10.1016/j.ymssp.2013.06.004.

Li, R., Sopon, P., and He, D. Fault features extraction for bearing prognostics. J Intell Manuf, 2012. 23(2):313-321. doi:10.1007/s10845-009-0353-z.

Lin, T. R., Kim, E., and Tan, A. C. A practical signal processing approach for condition monitoring of low speed machinery using peak-hold-down-sample algorithm. Mech. Syst. Sig. Process., 2013. 36(2):256270. doi:10.1016/j.ymssp.2012.11.003.

Muruganatham, B., Sanjith, M., Krishnakumar, B., and Murty, S. S. Roller element bearing fault diagnosis using singular spectrum analysis. Mech. Syst. Sig. Process., 2013. 35(1):150-166. doi:10.1016/j.ymssp.2012.08.019.

Niknam, S. A., Songmene, V., and Au, Y. J. The use of acoustic emission information to distinguish between dry and lubricated rolling element bearings in lowspeed rotating machines. Int. J. Adv. Manuf. Technol., 2013. 69(9-12):2679-2689. doi:10.1007/s00170013-5222-4.

Peled, R., Braun, S., and Zacksenhouse, M. A blind deconvolution separation of multiple sources, with application to bearing diagnostics. Mech. Syst. Sig. Process., $2005 . \quad$ 19(6):1181-1195. doi:10.1016/j.ymssp.2005.08.019.

Peng, Y., Dong, M., and Zuo, M. J. Current status of machine prognostics in condition-based maintenance: a review. Int. J. Adv. Manuf. Technol., 2010. 50(1):297-313. doi:10.1007/s00170-009-2482-0.
Qiu, H., Lee, J., Lin, J., and Yu, G. Wavelet filter-based weak signature detection method and its application on rolling element bearing prognostics. J. Sound Vibration, 2006. 289(4):1066-1090. doi:10.1016/j.jsv.2005.03.007.

Randall, R. B. and Antoni, J. Rolling element bearing diagnosticsa tutorial. Mech. Syst. Sig. Process., 2011. 25(2):485-520. doi:10.1016/j.ymssp.2010.07.017.

Sawalhi, N. and Randall, R. Simulating gear and bearing interactions in the presence of faults: Part I. The combined gear bearing dynamic model and the simulation of localised bearing faults. Mech. Syst. Sig. Process., 2008. 22(8):1924-1951. doi:10.1016/j.ymssp.2007.12.001.

Sawalhi, N. and Randall, R. Vibration response of spalled rolling element bearings: Observations, simulations and signal processing techniques to track the spall size. Mech. Syst. Sig. Process., 2011. 25(3):846870. doi:10.1016/j.ymssp.2010.09.009.

Shakya, P., Darpe, A. K., and Kulkarni, M. S. Bearing diagnosis using proximity probe and accelerometer. Measurement, 2016. 80:190-200. doi:10.1016/j.measurement.2015.11.029.

Siegel, D., Al-Atat, H., Shauche, V., Liao, L., Snyder, J., and Lee, J. Novel method for rolling element bearing health assessmenta tachometer-less synchronously averaged envelope feature extraction technique. Mech. Syst. Sig. Process., 2012. 29:362376. doi:10.1016/j.ymssp.2012.01.003.

SKF. Bearing lifetime calculator. (Link accessible October 2017). URL http://webtools3.skf.com/ BearingCalc/.

Yu, J. Bearing performance degradation assessment using locality preserving projections and Gaussian mixture models. Mech. Syst. Sig. Process., 2011. 25(7):2573-2588. doi:10.1016/j.ymssp.2011.02.006.

Zarei, J., Tajeddini, M. A., and Karimi, H. R. Vibration analysis for bearing fault detection and classification using an intelligent filter. Mechatronics, 2014. 24(2):151-157. doi:10.1016/j.mechatronics.2014.01.003.

Zhang, B., Sconyers, C., Byington, C., Patrick, R., Orchard, M. E., and Vachtsevanos, G. A probabilistic fault detection approach: Application to bearing fault detection. IEEE Trans. Ind. Electron., 2011. 58(5). doi:10.1109/TIE.2010.2058072. 